\title{
CMOS and DTMOS Sense Amplifier for SRAM Application
}

\author{
Komal $^{1}$, Dr. Neelam Rup Prakash ${ }^{2}$ \\ ME Student, ECE Department,PEC University of Technology, Chandigarh, India ${ }^{1}$ \\ Professor (Supervisor), ECE Department, PEC University of Technology, Chandigarh, India ${ }^{2}$
}

\begin{abstract}
In this paper comparison between CMOS and DTMOS amplifiers for SRAM application using 180nm technology is done. The tool used for simulation is Cadence Tool. The power dissipation varies with variation in supply voltage. The delay and average power dissipated for various values of power supply has been discussed and reported. The DTMOS sense amplifier is preferred at lower supply voltages.
\end{abstract}

Keywords: Power dissipation, delay, SRAM, DTMOS, CMOS, Sense Amplifier.

\section{INTRODUCTION}

In designing of memory cell, sense amplifiers are used to get lesser power dissipation and delay. Depending upon the performance various types of sense amplifiers are used in designing the memory. The delay should be very low so that it can be detected even if there is a small change on the bit lines due to the read and write operation in sense amplifier. [1] The various designs are compared based on delay and power consumption for different supply voltages. In this paper DTMOS and CMOS circuits are compared which has 3 transistor stages between VDD to GND. For DTMOS, the transistor's gate and substrate are connected together and due to the body effect, the threshold voltage can be changed dynamically in MOSFET. DTMOS is an excellent technique to get lesser delay. [2]

\section{CIRCUIT DESCRIPTION AND OPERATION}

\section{A. CMOS (Complementary MOSFET) Sense Amplifier}

In this circuit, there are 3 transistor stages between VDD to GND, and bitlines B and B' are cut off from the output nodes $\mathrm{O}$ and $\mathrm{O}^{\prime}$ as shown in Fig1 and its circuit diagram in Cadence tool is shown in Fig3. When SE =0, the transistors, MP1, MP4, MN5 and MN6 will be enabled, and MP5, MP6 will be disabled. Therefore, the transistors MP5 and MP6 will be isolated from the input signals coming from bit lines. Nodes 1 and 2 are connected to GND by MN5, MN6, so MN3, MN4 will be disconnected. The output nodes will be charged to the VDD by the transistors MP1, MP4. As MN3, MN4 are turned off, both the output nodes will hold the VDD level. [3][4] When SE=1, the transistors, MP5, MP6 will be enabled and MN5, MN6, MP1, MP4 will be disabled. The bit lines signals from the transistors MP5 and MP6 are given to the gates of transistors MN3 and MN4. The voltage difference between the gates of transistors MN3 and MN4 induces a drain-to-source current difference between them. This current difference will be converted and amplified to a voltage difference between output nodes $\mathrm{O}$ and $\mathrm{O}$ ' by the amplifier which consist of MP2, MP3, MN1 and MN2. The full swing voltage level will be available at the output nodes for a very short period of time. [3][4]

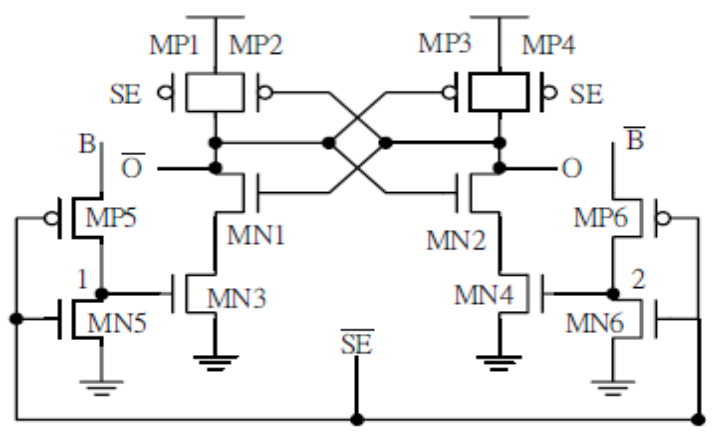

Fig1: CMOS Sense Amplifier

B. DTMOS (Dynamic Threshold Voltage MOSFET) Sense Amplifier

For DTMOS sense amplifier as shown in Fig2 and its circuit diagram in Cadence tool is shown in Fig4, the circuit and working is same as above CMOS sense amplifier. In DTMOS gate and substrate of the transistors are coupled together 


\section{ISO 3297:2007 Certified}

Vol. 5, Issue 6, June 2017

and due the body effect, the threshold voltage can be changed dynamically in MOSFET during the different modes of operation. When input given to the transistors is low, p-MOS is enabled with low $\mathrm{V}_{\text {th }}$ and n-MOS is disabled with normal $\mathrm{V}_{\text {th. }}$ In active mode due to the low $\mathrm{V}_{\text {th }} \mathrm{p}-\mathrm{MOS}$, the circuit get switched from low to high with a higher speed. In the standby mode, the subthreshold current of high $\mathrm{V}_{\text {th }}$ transistor decides the static leakage current which is smaller. When input to the transistors is 'high', the working is vice-versa. [2]

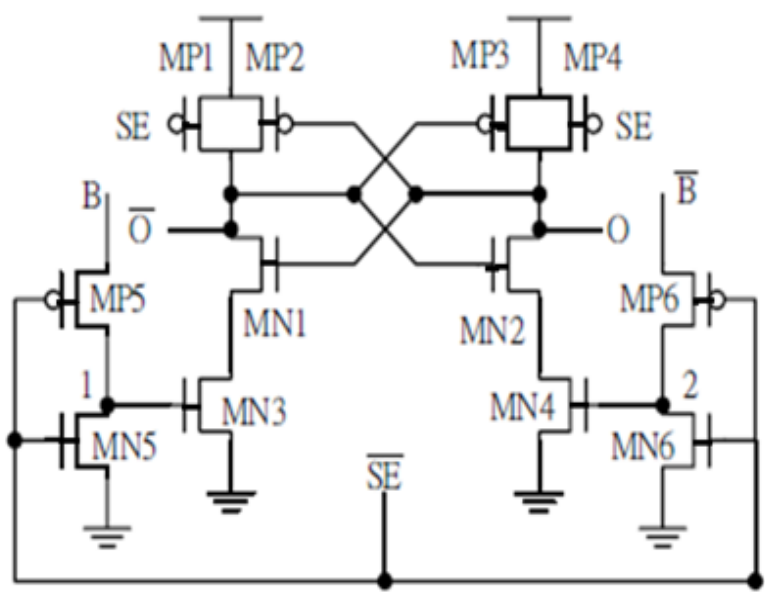

Fig2: DTMOS Sense Amplifier

\section{DESIGN AND SIMULATION RESULTS OF SENSE AMPLIFIER}

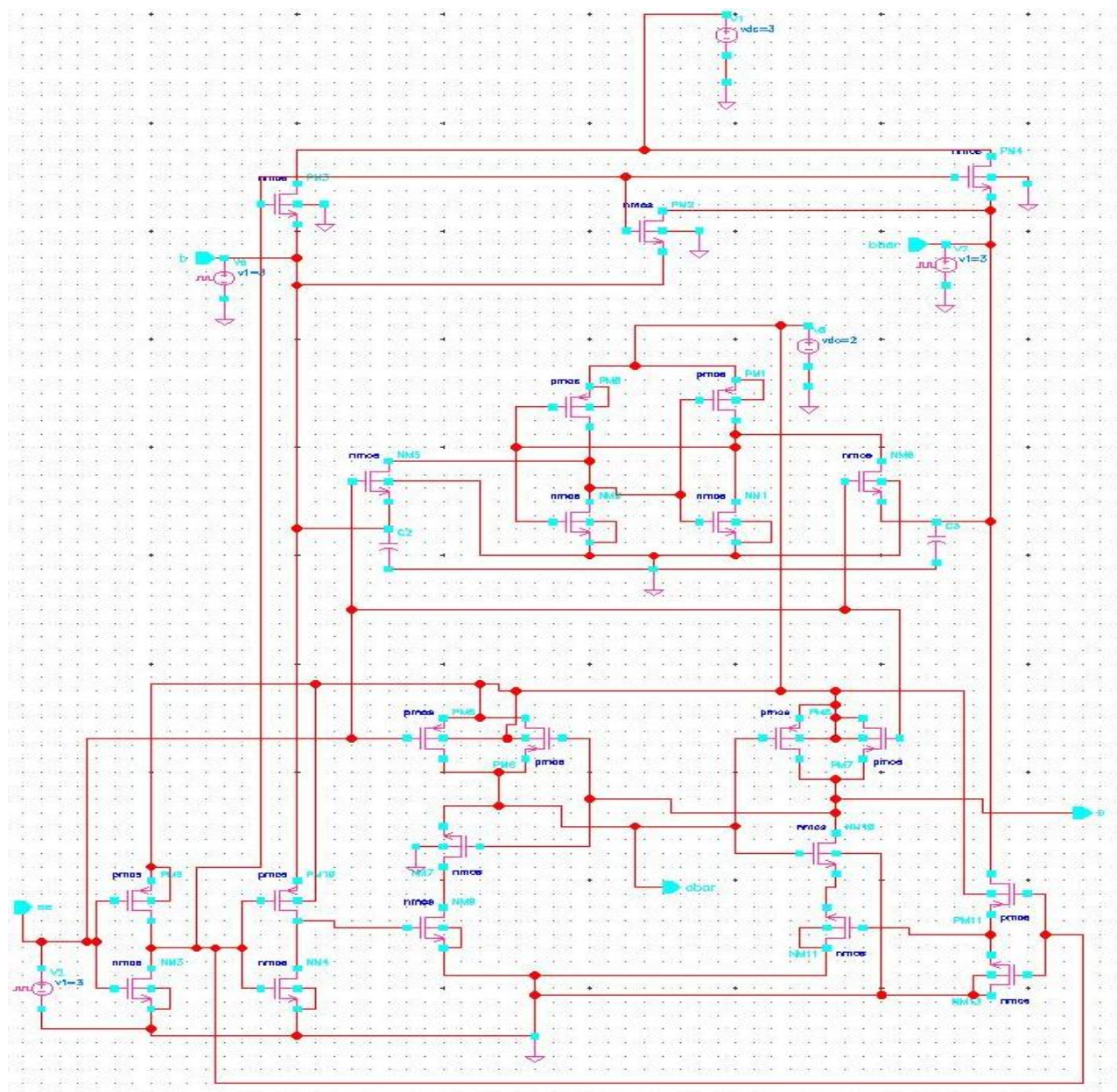

Fig3: Circuit Diagram of CMOS sense amplifier in Cadence Tool 
International Journal of Innovative Research in Electrical, Electronics, Instrumentation and Control Engineering

ISO 3297:2007 Certified

Vol. 5, Issue 6, June 2017

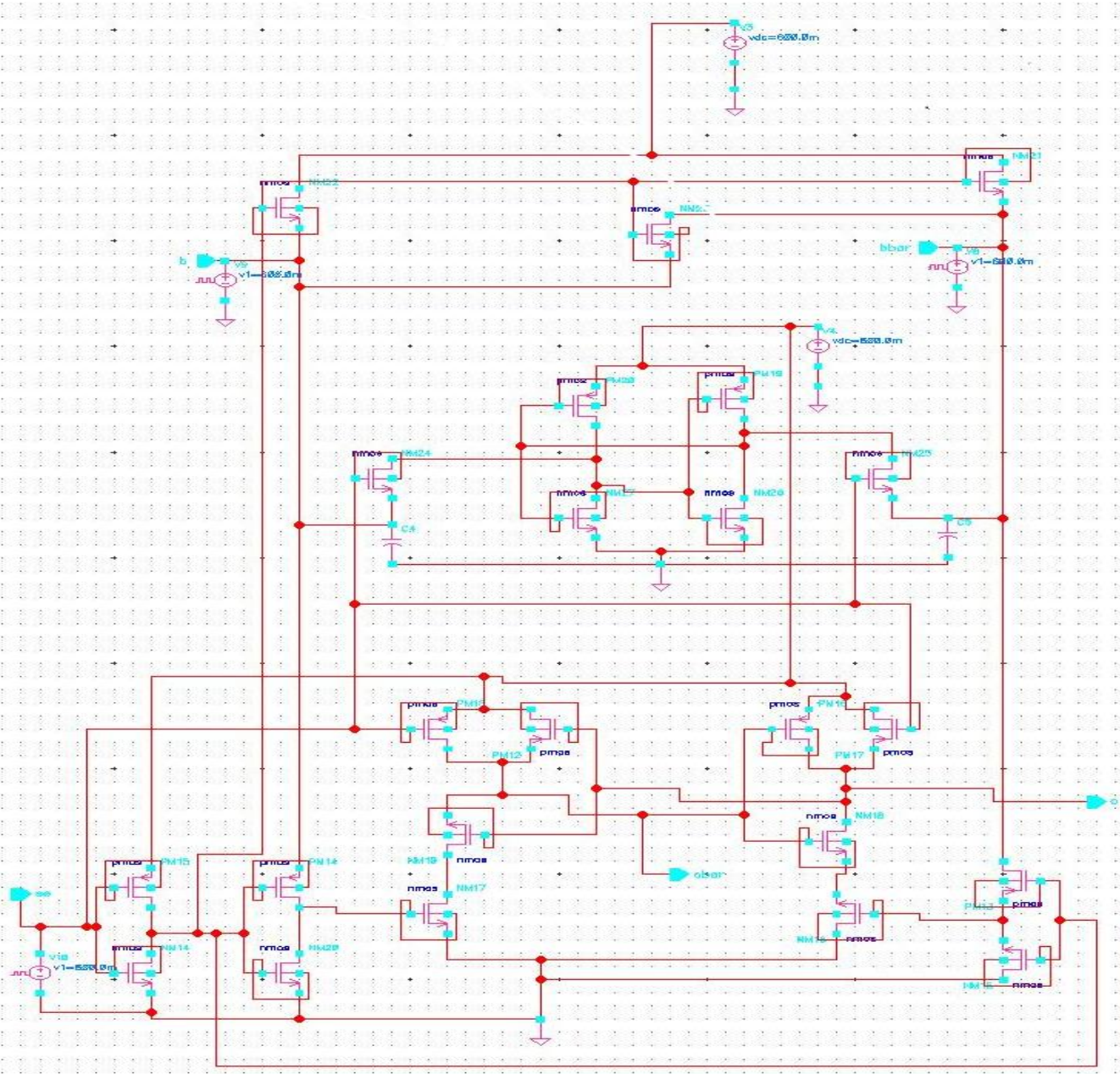

Fig4: Circuit Diagram of DTMOS sense amplifier in Cadence Tool

TABLEI: Variation of Power Consumed and Delay for CMOS Sense amplifiers

\begin{tabular}{|l|l|l|l|l|}
\hline S.NO. & VDD(V) & POWER(W) & DELAY(S) & POWER DELAY PRODUCT \\
\hline 1 & 0.3 & $6.678 * 10^{-9}$ & $225.3 * 10^{-9}$ & $1.50 * 10^{-15}$ \\
\hline 2 & 0.4 & $4.19 * 10^{-6}$ & $115.8 * 10^{-9}$ & $485.20^{*} * 10^{-15}$ \\
\hline 3 & 0.5 & $1.613 * 10^{-6}$ & $85.91 * 10^{-9}$ & $138.57 * 10^{-15}$ \\
\hline 4 & 0.6 & $12.37 * 10^{-6}$ & $27.72 * 10^{-9}$ & $342.90 * 10^{-15}$ \\
\hline
\end{tabular}

TABLEII: Variation of Power Consumed and Delay for DTMOS Sense amplifiers

\begin{tabular}{|l|l|l|l|l|}
\hline S.NO. & VDD(V) & POWER(W) & DELAY(S) & POWER DELAY PRODUCT \\
\hline 1 & 0.3 & $89.57 * 10^{-9}$ & $6.44 * 10^{-9}$ & $0.58 * 10^{-15}$ \\
\hline 2 & 0.4 & $1.206 * 10^{-6}$ & $40.04 * 10^{-9}$ & $48.29 * 10^{-15}$ \\
\hline 3 & 0.5 & $13.1 * 10^{-6}$ & $19.74 * 10^{-9}$ & $258.60^{*} 10^{-15}$ \\
\hline 4 & 0.6 & $128.8 * 10^{-6}$ & $17.22 * 10^{-9}$ & $2217.94 * 10^{-15}$ \\
\hline
\end{tabular}




\section{IJIREEICE \\ International Journal of Innovative Research in Electrical, Electronics, Instrumentation and Control Engineering \\ ISO 3297:2007 Certified \\ Vol. 5, Issue 6, June 2017}

\section{CONCLUSION}

In this paper CMOS and DTMOS sense amplifiers are compared based on power dissipation and delay as shown in table 1 and 2 respectively. The power dissipation decreases with decrease in supply voltage and minimum at 0.3 volt. From the results obtained, DTMOS sense amplifier has lower power delay product at lower supply voltages as compared to CMOS sense amplifier. So, it can be concluded that DTMOS sense amplifier is better than CMOS sense amplifier at lower supply voltage applications.

\section{REFERENCES}

[1] Hassan Siti Lailatul Mohd, Dayah Idalailah, Halim Ili Shairah Abdul, 2014, " Comparative study on 8T SRAM with different type of Sense Amplifier", IEEE-ICSE, Kuala Lumpur, Malaysia, pp321-324.

[2] Assaderaghi Fariborz, Sinitsky Dennis, Parke Stephen A., Bokor Jeffrey, Ko Ping K., and Hu Chenming, 1994, “A Dynamic Threshold-Voltage MOSFET (DTMOS) for very low Voltage Operation”, IEEE Electron Device Letters, Vol.15, No.12, pp 510-512.

[3] Chow Hwang-Cherng and Chang Shu-Hsien, 2004, "High Performance Sense Amplifier Circuit for Low Power SRAM Applications", ISCAS IEEE, pp741-744.

[4] Shalini, Kumar Anand, July-2013, “ Design of High Speed and Low Power Sense Amplifier for SRAM Applications”, IJSER, Volume 4, Issue 7, pp402-406 